\title{
Universal Index for Cirrhosis (UIC index): The development and validation of a novel index to predict advanced liver disease
}

This article was published in the following Dove Press journal:

Hepatic Medicine: Evidence and Research

\author{
Zohair Ahmed' \\ Jinma Ren² \\ Adam Gonzalez ${ }^{3}$ \\ Umair Ahmed ${ }^{4}$ \\ Saqib Walayat ${ }^{4}$ \\ Daniel K Martin ${ }^{5}$ \\ Harsha Moole ${ }^{4}$ \\ Sherri Yong 6 \\ Sean Koppe ${ }^{7}$ \\ Sonu Dhillon ${ }^{5}$ \\ 'Department of Gastroenterology \\ and Hepatology, University of Illinois \\ at Chicago, IL, USA; ${ }^{2}$ Department \\ of Center for Outcomes Research, \\ University of Illinois College of \\ Medicine, Peoria, IL, USA; '3niversity \\ of Illinois College of Medicine, Peoria, \\ IL, USA; ${ }^{4}$ Department of Internal \\ Medicine, University of Illinois \\ College of Medicine, Peoria, IL, USA; \\ ${ }^{5}$ Department of Gastroenterology \\ and Hepatology, University of Illinois \\ College of Medicine, Peoria, IL, USA; \\ ${ }^{6}$ Department of Pathology, University \\ of Illinois College of Medicine, Peoria, \\ IL, USA; 'Department of Hepatology, \\ University of Illinois at Chicago, IL, \\ USA
}

Correspondence: Zohair Ahmed Division of Gastroenterology and Hepatology, University of Illinois at Chicago, 840 South Wood Street, Suite 7/8E, Chicago, IL 606/2, USA

Tel + I 6306999247

Fax + I 3129965103

Email Zahmed23@uic.edu
Aim: The purpose of this study was to create and validate a novel serological diagnostic index to predict cirrhosis of all etiologies.

Methods: This was a retrospective observational study of 771 patients, age $>18$ years, who underwent a liver biopsy. The stage of fibrosis and routine laboratory values were recorded. The data were randomly separated into 2 datasets (training 50\% and testing 50\%). A stepwise logistic regression model was used to develop the novel index. The area under the curve of receiver operating characteristic (AUROC) was applied to compare the new index to existing ones (Fibro-Q, FIB4, APRI, AAR), which was also validated in the testing dataset.

Results: Variables associated with the presence of cirrhosis were first assessed by univariate analysis then by multivariable analysis, which indicated serum glutamic-oxaloacetic acid transaminase, serum glutamic-pyruvic transaminase, international normalized ratio, albumin, blood urea nitrogen, glucose, platelet count, total protein, age, and race were the independent predictors of cirrhosis $(P<0.05)$. Regression formula for prediction of cirrhosis was generated and a novel index was subsequently created. The diagnostic performance of the novel index for predicting cirrhosis was assessed using the receiver operating characteristic curve. The new index had significantly higher AUROC $(0.83,95 \%$ CI: $0.79-0.87)$ than Fibro-Q $(0.80,95 \%$ CI: $0.76-0.85)$, FIB4 (0.79, 95\% CI: 0.74-0.83), APRI (0.74, 95\% CI: 0.69-0.78), and AAR (0.72, 95\% CI: 0.67-0.78).

Conclusion: The novel index had the highest AUROC curve when compared with current indices and can be applied to all etiologies of chronic liver disease.

Keywords: cirrhosis, liver, fibrosis, diagnosis, screening, NAFLD, HCV, alcohol

\section{Introduction}

With over 25 million Americans affected, chronic liver disease (CLD) is a leading cause of morbidity and mortality in the USA. ${ }^{1-3}$ Identification of affected individuals early in the disease process remains the cornerstone of management and follow-up. Chronic liver inflammation leads to stellate cell activation with resultant fibrosis and eventual development of liver cirrhosis. Cirrhosis causes portal hypertension, and eventual onset of decompensation events: ascites, spontaneous bacterial peritonitis, varices, encephalopathy, and hepatocellular carcinoma. ${ }^{4-6}$ It is the assessment of this progression to cirrhosis that is critical to the development of each patient's health care plan. This is critical because survival in the compensated cirrhotic is $80 \%$ at 10 years, and after the first decompensating event, 5 -year survival falls to $15 \%{ }^{7}$ Thus, identification and treatment can significantly improve prognosis. 
Identification of such individuals remains a considerable diagnostic challenge. Traditionally, the liver biopsy has been the gold standard for determining diagnosis and prognosis of these individuals. In theory, this provides the ability to grade inflammation within the liver as well as stage of the disease, which is indicative of the level of fibrosis present. The main shortcomings with the biopsy include the invasive nature of the test, cost, inter-observer variability, inability to easily follow progression, and a sampling error up to $30 \% .{ }^{8}$

Recently, there has been extensive interest in finding reliable non-invasive methods to rapidly diagnose liver disease. Serological (APRI, FIB4, Forns Index, and Fibro Test) and radiological tests (ultrasound and MRI elastography) have been developed. ${ }^{9-14}$ The current noninvasive serological indices are limited: non-routine biomarkers are used, they predict cirrhosis only for specific etiologies, and when applied to all etiologies, their accuracy in predicting cirrhosis becomes quite poor. There has been a recent rise in mortality from CLD likely due to the obesity epidemic giving rise to increased cases of nonalcoholic fatty liver disease (NAFLD) and also due to baby boomers with hepatitis $\mathrm{C}$ virus (HCV) now presenting with acute decompensation events. ${ }^{15}$ Recognition of these individuals is especially important in the era of highly effective HCV therapy and molecular-targeted therapy, which can reverse fibrosis and even early cirrhosis. Although, the liver biopsy cannot be obviated, it may be augmented by a less invasive and equivalent if not more accurate screening and diagnostic test.

This need for more accurate identification of affected individuals led us to formulate a novel readily available index, which could be easily performed with routine laboratory measures in patients with chronic liver disease due to all etiologies. The novel Universal Index for Cirrhosis (UIC) was validated and compared with the presently available indices.

\section{Methods}

\section{Design and population}

This retrospective, observational study included 771 consecutive adult patients who had undergone percutaneous liver biopsies at Saint Francis Medical Center in Peoria, Illinois from April 2010 to October 2014. Patients with the following conditions were excluded from the study: presence of any type of cancer, non-liver disease, and insufficient liver tissue for staging of fibrosis. The analysis dataset, including 589 patients was randomly divided into 2 sets ( $50 \%$ training and $50 \%$ validation sets).

\section{Measure}

The primary outcome, cirrhosis, was defined as the stage 4 of fibrosis in this study. Batts and Ludwig scoring system was used to evaluate fibrosis stage, stage $0=$ no fibrosis, stage $1=$ portal fibrosis, stage $2=$ periportal fibrosis (including rare portal-portal septa), stage $3=$ septal fibrosis (with architectural distortion), and stage $4=$ bridging fibrosis and nodular regeneration. All results of liver biopsies were screened on hard-copy clinical charts by 2 reviewers separately.

Demographics and other clinical information were extracted from electronic medical record. We used the most recent values of serum markers within 90 days of liver biopsy, including serum glutamic oxaloacetic acid transaminase (SGOT), serum glutamic-pyruvic transaminase (SGPT), international normalized ratio (INR), blood urea nitrogen (BUN), creatinine, platelet count, total protein, alkaline phosphatase, total bilirubin, albumin and glucose.

\section{Ethical issue}

All study subjects had given consent for liver biopsy. This is a retrospective study where results would not change the course of patient care or current patient outcomes and all data were kept confidentially. Also, this study was approved by the institutional review board (IRB) at University of Illinois College of Medicine at Peoria and the need for patient informed consent was waived.

\section{Statistical analysis}

Statistical analysis was performed by SAS 9.4 (SAS Institute Inc., Cary, NC, USA). Frequency and percentage were used to describe the categorical variables, whereas mean and SD as well as histogram were applied to depict the continuous variables. The variables with skew distribution, such as SGOT, SGPT, BUN, BILI, and ALK, were analyzed after logarithmic transformation for normality. In order to cope with the missing values in some non-outcome variables $(<20 \%)$, multiple imputation technique was used to impute 10 output datasets, and combine their modeling results for the inference.

In view of the endpoint in this study was cirrhosis, a dichotomous variable, we performed a univariate logistic regression model on variables between patients with and without cirrhosis in the training set. Then, we created some new variables, such as $\log (\mathrm{SGOT}) / \log (\mathrm{SGPT}), \mathrm{INR} /$ albumin, and $\log (\mathrm{BUN}) / \log$ (glucose), because significant correlations among these variables were found. For the formulation of predictive model, multivariable analysis by stepwise logistic regression was used to identify independent factors associated with cirrhosis. 
New formula $\left(\mathrm{UIC}_{\text {index }}\right)$ with risk score that could best predict the cirrhosis was constructed based on the final regression model and the direction of effects. The 4 existing indices (Fibro-Q, FIB4, APRI, and AAR), (14) were also calculated in order to compare with the new formula. The diagnosis value of each formula was assessed by the area under the receiver operating characteristic (AUROC) curves. All models were tested for reliability in both the training set and the validation set based on the AUROC curves.

\section{Results}

\section{Patient characteristics}

Among 589 patients with liver biopsies, half of them (50.8\%) were male, average age was $53.4( \pm 11.8)$ years with a range of 20-91 years old. As Table 1 depicted, over one-third of them used alcohol (36\%) and/or tobacco (37.7\%) currently. The majority (79\%) were White American. The top etiology was Hepatitis C (42.9\%), followed by non-alcoholic steatohepatitis $(27.2 \%)$. The fibrosis of patients was across all stages $0-4$, but stages 2 and 4 accounted for over half.

\section{Development of a novel index for predicting cirrhosis}

Variables associated with the presence of cirrhosis were first assessed by univariate analysis (Table 2). Subsequent

Table I Profile of all patients

\begin{tabular}{lll}
\hline Items & Frequency & $\%$ \\
\hline $\begin{array}{l}\text { Gender } \\
\text { Male }\end{array}$ & 299 & 50.8 \\
Etiology & & \\
$\quad$ Hepatitis C & 253 & 42.9 \\
Non-alcoholic fatty liver disease & 160 & 27.2 \\
Alcoholic liver disease & 30 & 5.1 \\
Hepatitis B & 14 & 2.4 \\
Other liver diseases & 132 & 22.4 \\
Race/ethnicity & & \\
White American & 466 & 79.1 \\
Black American & 89 & 15.1 \\
Others & 34 & 5.8 \\
Alcohol use (yes) & 212 & 36.0 \\
Tobacco use & & \\
Yes, currently use & 222 & 37.7 \\
Already quit & 188 & 29.7 \\
Never use & 179 & 32.6 \\
Fibrosis stage & & \\
Stage 0 & 86 & 14.6 \\
Stage I & 84 & 14.3 \\
Stage 2 & 181 & 30.7 \\
Stage 3 & 69 & 11.7 \\
Stage 4 & 169 & 28.7 \\
Age (year), mean \pm SD & $53.4 \pm I I .8$ & \\
\hline
\end{tabular}

multivariable analysis indicated that $\log (\mathrm{SGOT}) / \log (\mathrm{SGPT})$, INR/albumin, $\log (\mathrm{BUN}) / \log$ (glucose), platelet count, total protein, age, and race were the independent predictors of cirrhosis $(P<0.05)$. Regression formula for prediction of cirrhosis was as under:

Risk score $=-7.52+3.18 \times \log (\mathrm{SGOT}) / \log (\mathrm{SGPT})+$ $4.99 \times \mathrm{INR} /$ albumin $-3.73 \times \log (\mathrm{BUN}) / \log ($ glucose $)-0.01 \times$ platelet $+0.74 \times$ total protein $+0.03 \times$ age- $1.46 \times$ race where race stands for Black American and others compared with White American.

According to the selected predictors and the direction of effects, we devised a novel index called UIC index as depicted in Box 1. The authors created a website, www. uicindex.com, where clinicians can plug in lab values and it will predict how likely or unlikely their patients have advanced liver disease.

Table 2 Univariate logistic regression in the training set

\begin{tabular}{|c|c|c|c|}
\hline Variables & $\begin{array}{l}\text { No cirrhosis } \\
\mathrm{n}=223\end{array}$ & $\begin{array}{l}\text { Cirrhosis } \\
n=71\end{array}$ & $P$-value \\
\hline Age, years & $52.52 \pm 12.16$ & $55.91 \pm 11.06$ & 0.059 \\
\hline Male gender, n (\%) & $116(52.0)$ & $29(40.9)$ & 0.102 \\
\hline \multicolumn{4}{|l|}{ Ethnicity, n (\%) } \\
\hline Black American/others & $56(25.1)$ & II (I5.5) & 0.096 \\
\hline White American & $167(74.9)$ & $60(84.5)$ & Ref. \\
\hline Alcohol use, n (\%) & $81(36.3)$ & $20(28.2)$ & 0.209 \\
\hline \multicolumn{4}{|l|}{ Tobacco use, n (\%) } \\
\hline Currently use & $94(42.2)$ & $24(33.8)$ & 0.488 \\
\hline Already quit & 64 (28.7) & $26(36.6)$ & 0.503 \\
\hline Never use & $65(29.1)$ & $21(29.6)$ & Ref. \\
\hline Body mass index & $29.62 \pm 7.49$ & $30.47 \pm 7.29$ & 0.182 \\
\hline \multicolumn{4}{|l|}{ Routine biomarkers } \\
\hline $\mathrm{SGOT}(\mathrm{U} / \mathrm{L})^{\mathrm{a}}$ & $4.09 \pm 0.77$ & $4.3 \pm 0.62$ & 0.082 \\
\hline SGPT(U/L) $)^{a}$ & $4.19 \pm 0.79$ & $4.08 \pm 0.72$ & 0.149 \\
\hline BUN $(\mathrm{mg} / \mathrm{dL})^{\mathrm{a}}$ & $2.65 \pm 0.44$ & $2.63 \pm 0.48$ & 0.236 \\
\hline Platelet count $\left(10^{9} / \mathrm{L}\right)$ & $220.07 \pm 75.8 I$ & $\mid 49.1 \pm 68.93$ & $<0.001$ \\
\hline $\mathrm{TP}(\mathrm{g} / \mathrm{dL})$ & $7.2 \pm 0.89$ & $7.16 \pm 0.91$ & 0.996 \\
\hline INR & $1.07 \pm 0.25$ & $1.24 \pm 0.32$ & $<0.001$ \\
\hline $\operatorname{ALK}(U / L)^{a}$ & $4.74 \pm 0.53$ & $4.83 \pm 0.4$ & $0.26 \mathrm{I}$ \\
\hline Bilirubin $(\mathrm{mg} / \mathrm{dL})^{\mathrm{a}}$ & $-0.37 \pm 0.85$ & $0.2 \pm 0.96$ & $<0.001$ \\
\hline Albumin (g/dL) & $3.91 \pm 0.62$ & $3.47 \pm 0.74$ & $<0.001$ \\
\hline Creatinine $(\mathrm{mg} / \mathrm{dL})^{\mathrm{a}}$ & $-0.15 \pm 0.4 \mid$ & $-0.13 \pm 0.45$ & 0.718 \\
\hline Glucose $(\mathrm{mg} / \mathrm{dL})^{\mathrm{a}}$ & $4.67 \pm 0.3$ & $4.73 \pm 0.32$ & 0.460 \\
\hline \multicolumn{4}{|l|}{ Created variables } \\
\hline SGOT_SGPT & $0.98 \pm 0.11$ & $1.07 \pm 0.14$ & $<0.001$ \\
\hline INR_Albumin ${ }^{c}$ & $0.29 \pm 0.14$ & $0.39 \pm 0.19$ & $<0.001$ \\
\hline BUN_Glucose ${ }^{d}$ & $0.57 \pm 0.09$ & $0.55 \pm 0.09$ & 0.091 \\
\hline
\end{tabular}

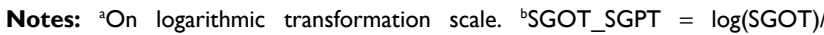

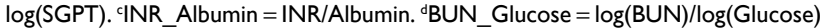
Abbreviation: BUN, blood urea nitrogen; INR, international normalized ratio; SGOT, serum glutamic-oxaloacetic acid transaminase; SGPT, serum glutamicpyruvic transaminase. 
Box I UIC index formula

$$
U I C_{\text {index }}=\frac{10 \times \text { Age } \times I N R \times \text { Total protein } \times \log (S G O T) \times \log (\text { glucose })}{\text { Albumin } \times \text { Platelet count } \times \text { race } \times \log (S G P T) \times \log (B U N)}
$$

where

\section{Age: year}

Total protein: gram/deciliter $(\mathrm{g} / \mathrm{dL})$

Platelet count: $10^{9} / \mathrm{L}$

SGOT and SGPT: units/liter (U/L)

Glucose, Albumin and BUN: milligram/deciliter (mg/dL)

Race: 1=White American, 2=Black American and others.

\section{Diagnostic performance of UIC ${ }_{\text {index }}$}

The diagnostic performance of the $\mathrm{UIC}_{\text {index }}$ for predicting cirrhosis was assessed using the ROC curve. As Figure 1 depicted, the AUROC of $\mathrm{UIC}_{\text {index }}$ for predicting cirrhosis were 0.82 (95\% CI: $0.75-0.87$ ) and 0.83 (95\% CI: $0.77-$ 0.88 ) for any etiology in the training and validation sets, respectively; were 0.87 (95\% CI: 0.78-0.94) and 0.84 (95\% CI: $0.75-0.92)$ for $\mathrm{HCV}$ in the training and validation sets, respectively.

The $\mathrm{UIC}_{\text {index }}$ was compared with 4 other published noninvasive indices (Fibro-Q, FIB4, APRI and AAR), which were available to be calculated in our data (Table 3 ). In the training set, the AUROC of $\mathrm{UIC}_{\text {index }}$ was significantly higher

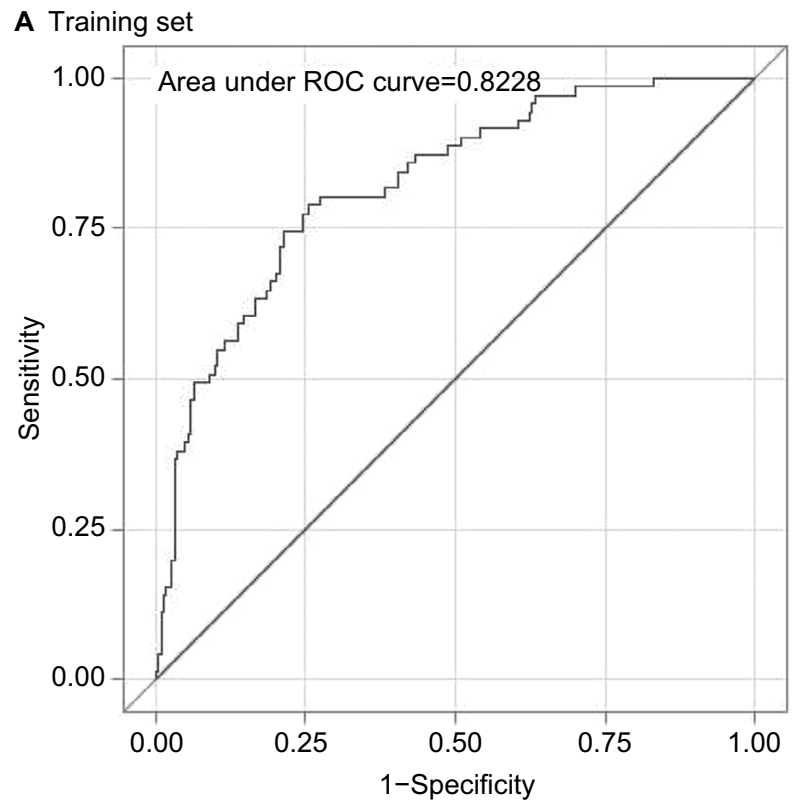

than FIB4 (0.77, 95\% CI: 0.71-0.84), APRI (0.71, 95\% CI: $0.64-0.78)$, and AAR $(0.73,95 \%$ CI: $0.66-0.80)$ when predicting cirrhosis in any etiology, and was significantly >AAR $(0.66,95 \%$ CI: $0.52-0.80)$ when predicting cirrhosis in $\mathrm{HCV}$ patients. The advantage of $\mathrm{UIC}_{\text {index }}$ was also demonstrated in the validation set even if the sample size

Table 3 Comparing the UIC $_{\text {index }}$ of cirrhosis with other existing indices

\begin{tabular}{|c|c|c|c|c|c|}
\hline & \multirow[t]{2}{*}{ Index } & \multicolumn{2}{|c|}{ Training set } & \multicolumn{2}{|c|}{ Validation set } \\
\hline & & AUROC & $95 \% \mathrm{Cl}$ & AUROC & $95 \% \mathrm{Cl}$ \\
\hline Any & Fibro-Q & 0.79 & $0.72-0.85$ & 0.82 & $0.76-0.87$ \\
\hline \multirow[t]{4}{*}{ etiology } & FIB4 & $0.77^{\mathrm{a}}$ & $0.7 I-0.84$ & 0.82 & $0.77-0.87$ \\
\hline & APRI & $0.7 I^{\mathrm{a}}$ & $0.64-0.78$ & 0.77 & $0.72-0.83$ \\
\hline & AAR & $0.73^{\mathrm{a}}$ & $0.66-0.80$ & $0.72^{\mathrm{a}}$ & $0.66-0.78$ \\
\hline & $\mathrm{UIC}_{\text {index }}$ & 0.82 & $0.75-0.87$ & 0.83 & $0.77-0.88$ \\
\hline \multirow[t]{5}{*}{ HCV only } & Fibro-Q & 0.79 & $0.68-0.90$ & 0.84 & $0.76-0.92$ \\
\hline & FIB4 & 0.82 & $0.72-0.92$ & 0.87 & $0.79-0.94$ \\
\hline & APRI & 0.77 & $0.65-0.88$ & 0.86 & $0.78-0.93$ \\
\hline & AAR & $0.66^{\mathrm{a}}$ & $0.52-0.80$ & $0.67^{\mathrm{a}}$ & $0.56-0.79$ \\
\hline & $U I C_{\text {index }}$ & 0.87 & $0.78-0.94$ & 0.84 & $0.75-0.92$ \\
\hline
\end{tabular}

Note: aSignificant difference $(P<0.05)$ compared to UIC $C_{\text {index }}$.

Abbreviations: AUROC, area under the receiver operating characteristic; $\mathrm{HCV}$, hepatitis C virus; UIC, Universal Index for Cirrhosis.

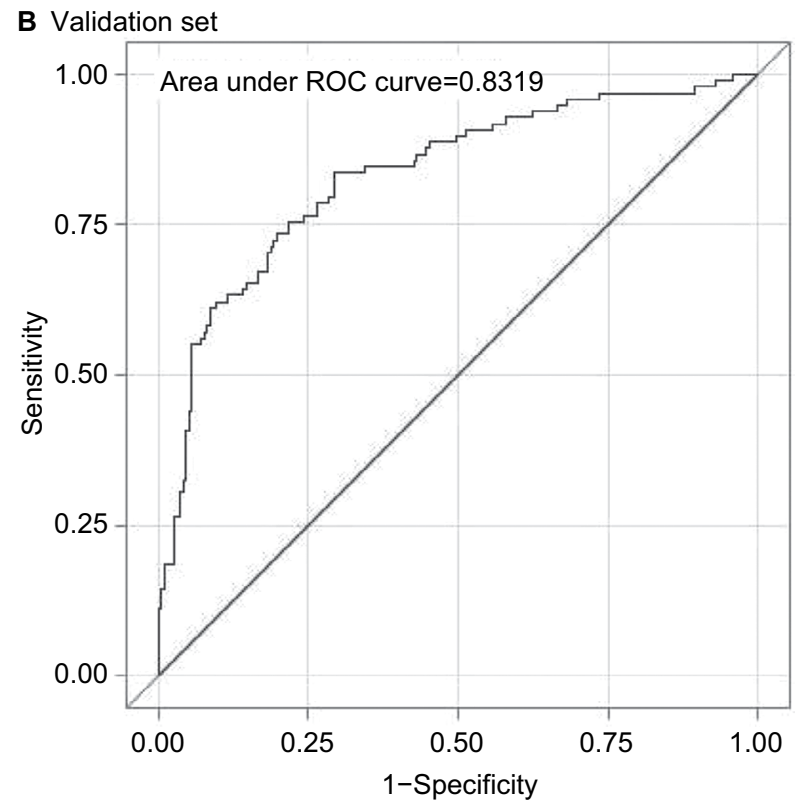

Figure I ROC curve of UIC index $_{\text {. }}$

Abbreviations: ROC, receiver operating characteristic; UIC, Universal Index for Cirrhosis. 
Table 4 Accuracy of the UIC $_{\text {index }}$ in predicting cirrhosis

\begin{tabular}{|c|c|c|c|c|c|c|c|}
\hline UIC $_{\text {index }}$ & $\begin{array}{l}\text { All patients } \\
\text { n (\%) }\end{array}$ & $\begin{array}{l}\text { No cirrhosis } \\
\text { (stage 0-3) } \\
\text { n (\%) }\end{array}$ & $\begin{array}{l}\text { Cirrhosis } \\
\text { (stage 4) } \\
\text { n (\%) }\end{array}$ & $\begin{array}{l}\text { NPV } \\
\%\end{array}$ & $\begin{array}{l}\text { PPV } \\
\%\end{array}$ & $\begin{array}{l}\text { Sensitivity } \\
\%\end{array}$ & $\begin{array}{l}\text { Positive and } \\
\text { negative likelihood } \\
\text { ratio }\end{array}$ \\
\hline \multicolumn{8}{|c|}{ Training set } \\
\hline$<4.5$ & $75(20)$ & $72(25)$ & $3(4)$ & 96.0 & 26.8 & 96.4 & 3.9 \\
\hline$\geq 4.5$ & $302(80)$ & $221(75)$ & $81(96)$ & & & & \\
\hline$<8.0$ & 177 (47) & $164(56)$ & $13(15)$ & 92.7 & 35.5 & 84.5 & 1.5 \\
\hline$\geq 8.0$ & $200(53)$ & $129(44)$ & 7I (85) & & & & \\
\hline$<20.0$ & $321(85)$ & $27 \mid(92)$ & $50(60)$ & 84.4 & 60.7 & 40.5 & 0.4 \\
\hline$\geq 20.0$ & $56(15)$ & $22(8)$ & $34(40)$ & & & & \\
\hline \multicolumn{8}{|c|}{ Validation set } \\
\hline$<4.5$ & $63(16)$ & $60(22)$ & $3(3)$ & 95.2 & 34.7 & 97.5 & 4.5 \\
\hline$\geq 4.5$ & $33 I(84)$ & $216(78)$ & II 5 (97) & & & & \\
\hline$<8.0$ & $173(44)$ & $158(57)$ & $15(13)$ & 91.3 & 46.6 & 87.3 & 1.5 \\
\hline$\geq 8.0$ & $221(56)$ & II 8 (43) & $103(87)$ & & & & \\
\hline$<20.0$ & $319(8 I)$ & $254(92)$ & $65(55)$ & 79.6 & 70.7 & 44.9 & 0.5 \\
\hline$\geq 20.0$ & $75(19)$ & $22(8)$ & $53(45)$ & & & & \\
\hline
\end{tabular}

Abbreviations: PPV, positive predictive value; NPV, negative predictive value; UIC, Universal Index for Cirrhosis.

was small. Overall, the $\mathrm{UIC}_{\text {index }}$ had a competent accuracy and robust compared with other indices.

\section{Cutoff values}

Based on the sensitivity and specificity of $\mathrm{UIC}_{\text {index }}, 3$ cutoff points $(4.5,8$, and 20$)$ were chosen to identify cirrhosis at different levels of predictive ability (Table 4). The presence of cirrhosis would be extremely likely if a patient's UIC $_{\text {index }}$ is $\geq 20$; in contrast, it would be extremely unlikely if the $\mathrm{UIC}_{\text {index }}$ is $<4.5$.

\section{Discussion}

CLD is a major cause of morbidity and mortality and is a major economic burden on the health care system. Together CLD and cirrhosis are the fifth leading cause of death in the 45-61 years age group and twelfth leading cause overall. ${ }^{1,2}$ Due to limitations of liver biopsy more reliable and less invasive modalities are constantly being sought. The use of non-invasive routine markers for diagnosis and staging of liver fibrosis and cirrhosis has recently garnered interest. ${ }^{16}$ Non-invasive indices are desirable due to their ability to provide an easily repeatable, inexpensive and highly applicable assessment of the liver. ${ }^{17}$ The most notable predictive models are: Fibro-Q, FIB4, APRI and AAR. While each of these models has their respective limitations, major issues with the majority of serum marker assessments, include the high level of heterogeneity between the etiologies as well as the use of non-routine markers, which are more difficult to obtain and are associated with increased cost. ${ }^{18-20}$

The main purpose of this research was to not only develop but also validate a novel index with the use of routine markers in the diagnosis of cirrhosis from all etiologies (NAFLD, $\mathrm{HCV}$, hepatitis B virus, and alcoholic liver disease). The diagnostic performance of the $\mathrm{UIC}_{\text {index }}$ as depicted in Figure 1 by the AUROC was 0.82 and 0.83 for any etiology in the training and validation sets, respectively; for HCV-only patients, it was 0.87 and 0.84 in the training and validation sets, respectively. The UIC index was superior in AUROC when compared with the current indices as depicted in Table 3, Fibro-Q: 0.82 vs 0.79; FIB4: 0.82 vs 0.77; APRI: 0.82

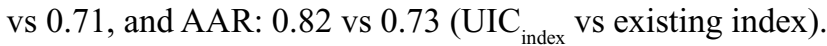

It is worth noting that race is one of parameters in our UIC index. Racial difference in the progression to cirrhosis has been reported in a previous study. ${ }^{21}$ They found that African Americans were at a considerably lower risk of developing cirrhosis than non-Hispanic White, and the association persisted even after adjusting a range of factors, including $\mathrm{HCV}$ genotype, HCV treatment, diabetes, and body mass index. Their findings of race are similar with the results in this study.

This study did have limitations. First of all, since more than one pathologist interpreted the liver biopsy, there may have been inter-observer variability in reported stage of fibrosis. Second, laboratory values done within 90 days of liver biopsy were included in the data set. Third, the ethnic majority was either Caucasian or African American; very few were of Hispanic or Asian descent.

The UIC index has the highest AUROC curve when compared with other indices and can be applied to all etiologies of CLD. The UIC index was also validated with similar results. It is our hope that clinicians utilize this index to rapidly identify affected individuals and are able to further validate this index. Patients with advanced liver disease may not be symptomatic 
and only come to medical attention when a decompensating event ensues. The UIC index will allow for rapid and early identification of at risk individuals and appropriate interventions may mitigate decompensation. Once identified, patients can be placed on highly effective anti-viral therapy for $\mathrm{HCV}$, be subject to strict diet/lifestyle modification for NAFLD, regular ultrasound examinations of hepatocellular carcinoma and endoscopic examinations of risky esophagogastric varices, and/or be placed as a liver transplant candidate.

\section{Acknowledgments}

The study was reviewed and approved by the University of Illinois, College of Medicine at Peoria IRB. An abstract of this paper has been presented at Digestive disease weekly conference in April 2016, American Association for the study of liver disease conference in November 2015, and American College of Gastroenterology meeting October 2016.

\section{Disclosure}

The authors report no conflicts of interest in this work.

\section{References}

1. Murphy SL, Xu J, Kochanek KD. Deaths: final data for 2010. Natl Vital Stat Rep. 2013;61(4):1-117.

2. Hoyert DL, Xu J. Deaths: preliminary data for 2011. Natl Vital Stat Rep. 2012;61(6):1-5.

3. Everhart JE. Digestive Diseases in the United States: Epidemiology and Impact. DIANE Publishing; 1994. Available from: https://books.google. co.nz/books?id=bQXwQ5u7yUQC\&dq=Everhart+JE. + Digestive + dise ases + in + the+United+States:+epidemiology+and+impact:+DIANE $+\mathrm{Pu}$ blishing,\&lr=\&source=gbs_navlinks_s. Accessed August 28, 2018.

4. Han YP. Matrix metalloproteinases, the pros and cons, in liver fibrosis. J Gastroenterol Hepatol. 2006;21(Suppl 3):S88-S91.

5. Bataller R, North KE, Brenner DA. Genetic polymorphisms and the progression of liver fibrosis: a critical appraisal. Hepatology. 2003;37(3):493-503.
6. Iredale JP. Models of liver fibrosis: exploring the dynamic nature of inflammation and repair in a solid organ. J Clin Invest. 2007;117(3): 539-548.

7. Mcmahon BJ. The natural history of chronic hepatitis B virus infection. Hepatology. 2009;49(5 Suppl):S45-S55.

8. Keltch B, Lin Y, Bayrak C. Comparison of AI techniques for prediction of liver fibrosis in hepatitis patients. J Med Syst. 2014;38(8):60.

9. Tapper EB, Castera L, Afdhal NH. FibroScan (vibration-controlled transient elastography): where does it stand in the United States practice. Clin Gastroenterol Hepatol. 2015;13(1):27-36.

10. Wong GL. Transient elastography: Kill two birds with one stone? World J Hepatol. 2013;5(5):264-274.

11. Chon YE, Choi EH, Song KJ, et al. Performance of transient elastography for the staging of liver fibrosis in patients with chronic hepatitis B: a meta-analysis. PLoS One. 2012;7(9):e44930.

12. Chen YP, Liang XE. Non-invasive assessment of liver fibrosis: reduce or substitute the need for liver biopsy? Liver Int. 2015;35(11): 2483.

13. Cabibi D, Calvaruso V, Giuffrida L, et al. Comparison of Histochemical Staining Methods and Correlation with Transient Elastography in Acute Hepatitis. Pathobiology. 2015;82(1):48-52.

14. Juárez-Hernández E, Uribe-Ramos MH, Ramos-Ostos MH, et al. Factors Associated with the Quality of Transient Elastography. Dig Dis Sci. 2015;60(7):2177-2182.

15. Koppe SW. Obesity and the liver: nonalcoholic fatty liver disease. Transl Res. 2014;164(4):312-322.

16. Abdollahi M, Pouri A, Ghojazadeh M, Estakhri R, Somi M. Non-invasive serum fibrosis markers: A study in chronic hepatitis. Bioimpacts. 2015;5(1):17-23.

17. Bourliere M, Penaranda G, Renou C, et al. Validation and comparison of indexes for fibrosis and cirrhosis prediction in chronic hepatitis $\mathrm{C}$ patients: proposal for a pragmatic approach classification without liver biopsies. J Viral Hepat. 2006;13(10):659-670.

18. Xu XY, Kong H, Song RX, et al. The effectiveness of noninvasive biomarkers to predict hepatitis B-related significant fibrosis and cirrhosis: a systematic review and meta-analysis of diagnostic test accuracy. PLoS One. 2014;9(6):e100182.

19. Soresi M, Giannitrapani L, Cervello M, Licata A, Montalto G. Non invasive tools for the diagnosis of liver cirrhosis. World J Gastroenterol. 2014;20(48):18131-18150.

20. Fallatah HI. Noninvasive Biomarkers of Liver Fibrosis: An Overview. Adv Hepatol. 2014;2014(5):1-15.

21. El-Serag HB, Kramer J, Duan Z, Kanwal F. Racial differences in the progression to cirrhosis and hepatocellular carcinoma in HCV-infected veterans. Am J Gastroenterol. 2014;109(9):1427-1435.
Hepatic Medicine: Evidence and Research

\section{Publish your work in this journal}

Hepatic Medicine: Evidence and Research is an international, peerreviewed, open access journal covering all aspects of adult and pediatric hepatology in the clinic and laboratory including the following topics: Pathology, pathophysiology of hepatic disease; Investigation and treatment of hepatic disease; Pharmacology of drugs used for the treatment of hepatic disease. Issues of patient safety and quality of care will also be considered. The manuscript management system is completely online and includes a very quick and fair peer-review system, which is all easy to use. Visit http://www.dovepress.com/testimonials.php to read real quotes from published authors. 